\title{
Verbleib der Absolventinnen und Absolventen der Modellstudiengänge in den Gesundheitsfachberufen in Nordrhein- Westfalen: Ergebnisse zu Beschäftigungsmerkmalen und Kompetenzen in der Berufspraxis
}

\author{
Graduates of the Model Study Courses in the Health Professions in \\ North Rhine-Westphalia: Employment Characteristics and \\ Competencies in Professional Practice
}

\section{(๑) $\odot \Theta$}

\author{
Autoren \\ Sven Dieterich'미, Christian Grebe ${ }^{2}$, Christoph Bräutigam ${ }^{3}$, Rüdiger Hoßfeld ${ }^{4}$, Änne-Dörte Latteck ${ }^{2}$, Anke Helmbold ${ }^{5}$, \\ Stefan Heim ${ }^{6}$, Marcellus Bonato ${ }^{7}$, Jörg große Schlarmann ${ }^{8}$, Renate Adam-Paffrath ${ }^{9}$, Sascha Sommer ${ }^{1}$, Elke Oetken ${ }^{10}$, \\ Nina Jacobs ${ }^{6}$, Anke Mijatovic 5
}

Institute

1 Department für Angewandte Gesundheitswissenschaften, Hochschule für Gesundheit Bochum, Bochum

2 Fachbereich Wirtschaft und Gesundheit, Fachhochschule Bielefeld, Bielefeld,

3 Forschungsschwerpunkt Arbeit und Wandel, Institut Arbeit und Technik der Westfälischen Hochschule, Gelsenkirchen, Bocholt, Recklinghausen

4 Stabsstelle Qualität in Studium und Lehre, Hochschule für Gesundheit Bochum, Bochum

5 Fachbereich Gesundheitswesen, Katholische Hochschule Nordrhein Westfalen, Köln

6 Medizinische Fakultät, RWTH Aachen University, Aachen

7 Fachbereich Gesundheit, Münster, Fachhochschule Münster

8 Hochschulbereich Gesundheit, praxisHochschule - Standort Rheine, Rheine

9 Lehrgebiet Pflege und Gesundheit, Fliedner Fachhochschule Düsseldorf, Düsseldorf

10 Schule für Logopädie, Uniklinik RWTH Aachen, Aachen

online publiziert $\quad 13.10 .2020$

Bibliografie

Gesundheitswesen 2020; 82: 920-930

DOI 10.1055/a-1241-3983

ISSN $0941-3790$

(C) 2020. The Author(s).

This is an open access article published by Thieme under the terms of the Creative Commons Attribution-NonDerivative-NonCommercial-License, permitting copying and reproduction so long as the original work is given appropriate credit. Contents may not be used for commercial purposes, or adapted, remixed, transformed or built upon. (https://creativecommons.org/licenses/by-nc-nd/4.0/)

Georg Thieme Verlag KG, Rüdigerstraße 14, 70469 Stuttgart, Germany
Korrespondenzadresse

Sven Dieterich

Department für Angewandte Gesundheitswissenschaften Hochschule für Gesundheit Bochum

Gesundheitscampus 6-8

44801 Bochum

Deutschland

sven.dieterich@hs-gesundheit.de

\section{ZUSAMMENFASSUNG}

Ziel Ziel des Artikels ist es, zentrale Ergebnisse aus der landesweiten Studie zum Verbleib der Absolventen aus den $12 \mathrm{Mo-}$ dellstudiengängen in den Gesundheitsfachberufen in Nordrhein-Westfalen zu referieren. Untersuchungsziel der Studie ist es, den beruflichen Verbleib nach dem Abschluss eines berufsqualifizierenden Modellstudiums zu beschreiben und Merkmale sowie Rahmenbedingungen der beruflichen Situation darzustellen. Im Mittelpunkt des Artikels stehen Erkenntnisse zu den Merkmalen der Haupterwerbstätigkeit der Absolventen und zur Anwendung der im Studium vermittelten Kompetenzen im Berufsalltag.

Methodik Insgesamt 515 Absolventen aus den Modellstudiengängen Pflege ( $N=244)$, Physiotherapie $(N=97)$, Logopädie $(\mathrm{N}=95)$, Ergotherapie $(\mathrm{N}=47)$ und Hebammenkunde $(\mathrm{N}=32)$ haben sich im Frühsommer 2018 an einer Online-QuerschnittBefragung beteiligt. In die Befragung eingeschlossen waren alle Abschlussjahrgänge zwischen Wintersemester 2013/2014 und Sommersemester 2017. Ergänzend wurden Arbeitgeber $(\mathrm{N}=109)$ befragt, die Absolventen aus den genannten Studiengängen eingestellt haben.

Ergebnisse Die Absolventen sind v. a. im Krankenhaus und in ambulanten Therapiepraxen tätig. Acht von 10 Absolventen (84\%) erbringen vornehmlich klientennahe Aufgaben. $71 \%$ 
dieser klientennah arbeitenden Absolventen erbringen im Vergleich zu fachschulisch ausgebildeten Personen ergänzend besondere Aufgaben. Die Absolventen schätzen sich in allen untersuchten Kompetenzdimensionen durchschnittlich als handlungssicher ein. Die Arbeitgeber nehmen in allen Kompetenzdimensionen bei den Absolventen einen „Kompetenzvorsprung“ im Vergleich zu fachschulisch Ausgebildeten wahr. Schlussfolgerungen Die Resultate der Verbleibstudie unterstützen die Empfehlung, die Studiengänge aller untersuchten Gesundheitsfachberufe berufsgesetzlich zu verankern und ihren Regelbetrieb an den Hochschulen einzuführen.

\section{ABSTRACT}

Aim The aim of this article was to present key results of the graduates' survey VAMOS. The study examined the professional status of the graduates of 12 model study courses in the health professions in North Rhine-Westphalia. The focus of the article was on the employment characteristics of the current main jobs and the application of the academic competencies in everyday professional life.
Methods In the early summer of 2018 (April to June), 515 graduates of the model study courses in nursing $(N=244)$, physiotherapy $(N=97)$, speech therapy $(N=95)$, occupational therapy $(\mathrm{N}=47)$, and midwifery $(\mathrm{N}=32)$ were included in an online cross-sectional survey. Graduating classes between the winter semester 2013/2014 and the summer semester 2017 were included. In addition, employers ( $N=109)$ were interviewed who hired graduates from the model study courses.

Results The main jobs of the graduates were mostly located in hospitals and outpatient therapy practices. Eight out of ten graduates $(84 \%)$ performed tasks in direct contact with patients as the main part of their employment. In $71 \%$ of these cases, the regular tasks were combined with extended activities in comparison to colleagues without an academic degree. On average, the graduates felt confident in all competency dimensions examined in this study. In all dimensions, employers perceived a "competence advantage" for graduates compared to colleagues without an academic degree.

Conclusion The results of this graduate survey support the current recommendation to have the study programs in the five health care professions governed by professional laws and to enable the programs to be carried out at universities.

\section{Einleitung}

Der Versorgungsbedarf einer ansteigenden Zahl chronisch und mehrfacherkrankter Menschen bedingt anspruchsvollere Anforderungsprofile in den Gesundheitsfachberufen [1,2]. Das Fachpersonal in Pflege, Hebammenwesen, Physiotherapie, Logopädie und Ergotherapie ist aufgefordert, interprofessionell und sektorenübergreifend mit anderen Berufsgruppen zusammenzuarbeiten [1,3]. Hinzu kommt die Notwendigkeit, evidenzbasiert zu handeln und Beratungsaufgaben vor dem Hintergrund steigender Informationsbedürfnisse der Klienten/Patienten wahrzunehmen [1]. Die hochschulische Erstqualifizierung, wie sie im europäischen Ausland weitgehend üblich ist, wird daher auch in Deutschland als Element zukunftsfähiger Ausbildungsstrukturen angesehen, um den veränderten Anforderungen in den Gesundheitsfachberufen gerecht zu werden $[1,4]$.

Als Folge von Erprobungsregelungen (Modellklauseln), die der Gesetzgeber in den Berufsgesetzen der Pflege [5, 6], der Therapieberufe sowie der Hebammenkunde [7] geschaffen hat, ist die hochschulische Erstqualifizierung auch in Deutschland möglich geworden. In Nordrhein-Westfalen (NRW) wurden seit Wintersemester 2010/2011 an 7 Hochschulen 12 berufsqualifizierende Modellstudiengänge angeboten. Fünf der Studiengänge hatten die Fachrichtung Pflege. Darüber hinaus wurden 3 Logopädie- und 2 Physiotherapiestudiengänge sowie jeweils einer mit den Fachrichtungen Ergotherapie und Hebammenkunde angeboten. Gemeinsames Merkmal der Studiengänge ist, dass die Gesamtverantwortung für die Berufsqualifizierung bei den Hochschulen und nicht bei Berufsfachschulen liegt. Im Rahmen der Modellstudiengänge erwerben Studierende einen staatlichen Berufs- und einen Bachelorabschluss. Ein Qualifikationsmehrwert im Vergleich zur fachschulischen Ausbildung ist hierbei in der curricularen Konzeption der Studiengänge zu sehen. Die Hochschulcurricula greifen gezielt die veränder- ten Anforderungen der Versorgung auf, indem sie u. a. auf „die Anwendung wissenschaftlichen Wissens“ im Kontext klinischen Handelns, die „Fähigkeit zum Aufbau und zur Reflexion von Arbeitsbündnissen“ und die „Förderung von interprofessionellem Lernen und Handeln" als wesentliche Kompetenz- und Bildungsziele fokussiert sind [8]. Ziel ist es, berufsqualifizierende Studiengänge anzubieten, die zu beruflicher Handlungskompetenz befähigen, um pflegerische, therapeutische oder hebammenkundliche Prozesse in allen Bereichen der gesundheitlichen Versorgung und unter Berücksichtigung aktueller wissenschaftlicher Evidenz durchführen und reflektieren zu können. Als Einsatzbereich für die Absolventen werden v. a. „komplexe Aufgabenbereiche“ in der direkten Versorgung von Klienten/Patienten gesehen [1]. Die Evaluation der Modellstudiengänge in NRW im Jahre 2014 belegte eine erfolgreiche Kompetenzvermittlung und zeigte den Bedarf für die Untersuchung von Fragen zum beruflichen Verbleib der Studienabsolventen auf [9].

Um Aussagen zum Absolventenverbleib treffen zu können, wurde durch das Ministerium für Arbeit, Gesundheit und Soziales die Verbleibstudie VAMOS gefördert (Projektlaufzeit: 2017-2019) [10]. Die Studie wurde ausgeführt von den 7 Hochschulen mit Modellstudiengängen, dem Institut für Bildungs- und Versorgungsforschung im Gesundheitsbereich (InBVG), dem Institut Arbeit und Technik (IAT) und dem Institut Arbeit und Qualifikation (IAQ).

Bisher haben sich bundesweit 3 empirische Untersuchungen mit der Frage des beruflichen Verbleibs von Absolventen aus Modellstudiengängen beschäftigt. Daten von Absolventen aus NRW sind hierbei lediglich in der Untersuchung von Baumann und Kugler enthalten, die insgesamt 273 Pflegeabsolventen aus 14 Hochschulen im gesamten Bundesgebiet zum Verbleib und zur Zufriedenheit mit der beruflichen Arbeitssituation befragt haben [11]. In einer Online-Befragung des Hochschulverbunds Gesundheitsfach- 
berufe (HVG) wurden zudem 143 Absolventen aus therapeutischen Modellstudiengängen außerhalb des Bundeslands NRW nach ihrer Berufstätigkeit und Berufszufriedenheit befragt $[12,13]$. Für die Berufsgruppe der Hebammen ist in einer qualitativen Studie der berufliche Verbleib der ersten Absolventenkohorte der hessischen Hochschule Fulda beschrieben worden $(\mathrm{N}=8)$ [14].

Mit den Ergebnissen der Studie VAMOS liegen erstmals umfangreiche Daten für alle Berufsgruppen und Modellstudiengänge in NRW aus Absolventen- und Arbeitgeberperspektive vor [10]. Im vorliegenden Artikel werden ausgewählte quantitative Ergebnisse geschildert, die einen Beitrag zur Beurteilung der Studiengänge im Hinblick auf zentrale Beschäftigungsmerkmale und Bildungsparameter in Form des Einbringens der Kompetenzen in die Versorgung liefern können. Leitend waren hierbei folgende Fragestellungen [10]:

\section{Arbeitsfelder und Aufgabenbereiche}

1. In welchen Arbeitsfeldern bzw. Settings arbeiten die Absolventen?

2. Übernehmen die Absolventen besondere Aufgabenbereiche und, falls ja, um welche handelt es sich dabei?

\section{Ausprägung und Nutzung der Kompetenzen}

3. Wie schätzen die Absolventen selbst ihr Kompetenzniveau ein?

4. In welchem Maße bringen die Absolventen ihre erworbenen Kompetenzen in ihre berufliche Praxis ein?

5. Wie werden die spezifischen Kompetenzen der hochschulisch qualifizierten Absolventen von Seiten der Arbeitgeber eingeschätzt und eingesetzt?

\section{Methodik}

\section{Befragung der Absolventen}

Die Perspektive der Absolventen wurde mittels einer als standardisierte Onlinebefragung angelegten Querschnittserhebung im Zeitraum von April bis Juni 2018 erhoben. Die Zielpopulation umfasste alle 1124 Studierenden der 12 Modellstudiengänge in NRW mit Studienabschluss bis spätestens Sommersemester 2017. Die Befragten erhielten von ihren Hochschulen individuelle Zugangscodes zur Teilnahme an der Befragung. Für die Datenerhebung wurde LimeSurvey in Version 2.73 eingesetzt. Die Datenanalyse erfolgte mit SPSS in Version 24 sowie in $R$.

Zur Erfassung der Handlungssicherheit in beruflichen Situationen wurden, ausgehend von den konsentierten „gemeinsamen Qualifizierungszielen“ der Modellstudiengänge [15], mit den Health Professionals Competence Scales (HePCoS) reliable und konstruktvalide Messinstrumente entwickelt. Diese Skalen weisen eine interne Konsistenz (Cronbach's Alpha) zwischen 0,79 und 0,95 auf [16]. Jede Skala erfasst mit einem Punktwert zwischen 0 und 100 die Ausprägung der Handlungssicherheit als Selbsteinschätzung in jeweils einer von acht Kompetenzdimension. Zudem wurde die Einschätzung der Kompetenznutzung der Absolventen erfragt (in Anlehnung an [17]).

Aufgabenprofile der Absolventen wurden in einem induktiven Verfahren aus verschiedenen Angaben (Klientennähe der Tätigkeit, Setting, besondere über die Aufgaben der Berufskollegen hinausgehende Aufgaben, angestellte vs. selbstständige Tätigkeit, Wahrnehmung einer Vorgesetztenfunktion, erforderliches Qualifikationsniveau der Tätigkeit, Tätigkeitsbeschreibung als Freitext) entwickelt und die Pro- file den Befragten anschließend deduktiv durch 2 unabhängige Personen zugeordnet. Vorwiegend klientennahe Erwerbstätigkeiten wurden dabei in 2 Profile untergliedert. Zum einen in solche, die sich hinsichtlich der Aufgabenbereiche nicht oder nur unwesentlich von den Aufgaben fachschulisch qualifizierter Kollegen unterscheiden. Zum anderen in solche Erwerbstätigkeiten, bei denen der geringere Teil der Tätigkeit aus besonderen Aufgaben besteht, die im Vergleich zu den jeweiligen Fachkollegen in höherem Maße übernommen werden (z. B. klinisch-fachliche Expertise, Prozesssteuerung, Beratung, Schnittstellenmanagement, wissenschaftliche Recherche, Projektarbeit, Qualitätsmanagement, Konzeptentwicklung, Leitungsaufgaben).

$\mathrm{N}=518$ Absolventen loggten sich in die Onlinebefragung ein, von denen 3 die Befragung bereits vor der ersten Frage abbrachen. In die Analyse flossen somit N=515 Absolventen ein. Von diesen entfielen 244 auf die Pflege, 97 auf die Physiotherapie, 95 auf die Logopädie, 47 auf die Ergotherapie und 32 auf die Hebammenkunde. Der Bruttorücklauf betrug 45,8\%. Die Rücklaufquote lagje nach Studienrichtung zwischen 40,0\% (Hebammenkunde) und 53,7\% (Physiotherapie). Die Verteilung der Abschlussjahrgänge unterschied sich in keiner der Studienrichtungen signifikant ( $p>0,05, C^{2}{ }^{2}$-Tests) von der Grundgesamtheit. Im arithmetischen Mittel ( \pm Standardabweichung) hatten die Absolventen ihr Bachelorstudium 1,9 $\pm 1,1$ Jahre vor dem Befragungszeitpunkt abgeschlossen. Die Abschlussnoten in der Stichprobe unterschieden sich signifikant um 0,1 Notenpunkte von jenen der Grundgesamtheit in der Pflege $(p=0,001)$ und in der Physiotherapie ( $p=0,029$, jeweils MannWhitney-U-Tests), nicht jedoch in den übrigen 3 Studienrichtungen.

\section{Befragung der Arbeitgeber}

Die Erfassung der Perspektive der Arbeitgeber erfolgte mittels einer standardisierten Online-Befragung, die von Juli bis Dezember 2018 durchgeführt wurde. Die Entwicklung des Erhebungsinstruments erfolgte auf Basis einer Literaturrecherche, die zum einen Interessen, Anforderungen und Erwartungen von Arbeitgebern im Kontext der Beschäftigung akademisch qualifizierter Mitarbeiter in Gesundheitsfachberufen und zum anderen fördernde und hemmende Faktoren im Einstellungs-, Einmündungs- und Entwicklungsprozess von Absolventen zum Gegenstand hatten. Der Fragebogen gliederte sich in mehrere Themenfelder mit jeweils mehreren Items und fragte die Einschätzungen der Arbeitgeber überwiegend mittels Rating-Skalen (bspw. „trifft voll zu“ bis „trifft überhaupt nicht zu“) ab. Das Instrument war für alle Berufe mit Ausnahme einiger Formulierungen (insb. Berufsbezeichnungen) einheitlich. Für die Datenerhebung wurde die Software SoSci Survey genutzt.

Die Befragung der Arbeitgeber beschränkte sich auf jene Einrichtungen und Unternehmen, die Absolventen der Modellstudiengänge in NRW eingestellt hatten. In der Befragung der Absolventen (s. o.) wurden die Teilnehmenden gebeten, anonym ihre Arbeitgeber zu nennen. Auf dieser Basis wurden per Internetrecherche die jeweils passenden Ansprechpartner sowie deren Telefonnummern und EMail-Adressen zusammengestellt. Lediglich etwa $20 \%$ der Absolventen machten Angaben zu Arbeitgebern. Daher wurde ergänzend auf Informationen der beteiligten Hochschulen/Modellstudiengänge über die beim Studium kooperierenden Praxiseinrichtungen sowie auf ergänzende Informationsquellen zurückgegriffen. Potenzielle Befragungsteilnehmer wurden telefonisch kontaktiert und über das Vorhaben informiert. Konnte der Arbeitgeber die Beschäftigung von Studienabsolventen bestätigen und äußerte die Bereitschaft zur Teil- 
nahme, wurde ein Link zum Fragebogen per E-Mail verschickt. Insgesamt beteiligten sich 109 Arbeitgeber, die zu mehr als der Hälfte aus dem Berufsfeld der Pflege stammten, während die 4 weiteren Berufe Anteile zwischen etwa 10 bis $14 \%$ erreichten. Bei den Institutionsarten dominierten das Krankenhaus, das durch mehr als 6 von 10 Befragten repräsentiert wurde, sowie die (Therapie)Praxis mit mehr als einem Viertel am Gesamtrücklauf. Aufgrund der geringen Zahl der einbezogenen Arbeitgeber wird eine Ergebnisdarstellung nicht differenziert nach Berufen vorgenommen. Die Datenanalyse erfolgte mit der Software SPSS.

Der Umgang mit den erhobenen Daten erfolgte sowohl in der Befragung der Absolventen als auch der Arbeitgeber nach vorheriger datenschutzrechtlicher Prüfung durch die Datenschutzbeauftragten der beteiligten Institute und Hochschulen.

\section{Ergebnisse}

\section{Arbeitsfelder und Aufgabenbereiche der Absolventen}

Ein Teil der Absolventen gab an, zum Befragungszeitpunkt zugleich mehreren Erwerbstätigkeiten nachzugehen (etwaiges weiteres Stu- dium nicht mitgerechnet). Dies ist am häufigsten unter den Hebammen (40\% der Absolventen) und in der Physiotherapie (31,5\%) der Fall, seltener in der Pflege (20\%), Logopädie (14\%) und Ergotherapie (12\%). Im Folgenden beziehen sich Angaben zu Beschäftigungsverhältnissen bzw. selbstständigen Erwerbstätigkeiten jeweils ausschließlich auf die Haupterwerbstätigkeit, womit jene mit den meisten Wochenarbeitsstunden gemeint ist.

93,3\% der Hebammen, 92,9\% der Ergotherapeuten, 86,9\% der Logopäden, 86,5\% der Physiotherapeuten sowie 78,0\% der Pflegenden üben Haupterwerbstätigkeiten aus, die vorwiegend durch klientennahe Aufgaben gekennzeichnet sind ( $>$ Abb. 1). In allen 5 Berufsgruppen handelt es sich dabei deutlich überwiegend um Tätigkeiten, bei denen die klientennahen Aufgabenbereiche zusätzlich um „besondere“ Aufgabenbereiche ergänzt werden, die durch die Absolventen in einem stärkeren Maße als durch deren Fachkollegen übernommen werden. Unter den vorwiegend klientennah arbeitenden Absolventen ist dies bei 84,6\% der Ergotherapeuten, bei 80,6\% der Logopäden, bei 75,0\% der Hebammen, bei 72,7\% der Physiotherapeuten und bei 62,9\% der Pflegenden der Fall.

Wissenschaftliche Tätigkeiten und wissenschaftliche Assistenztätigkeiten finden sich unter den Haupterwerbstätigkeiten in der

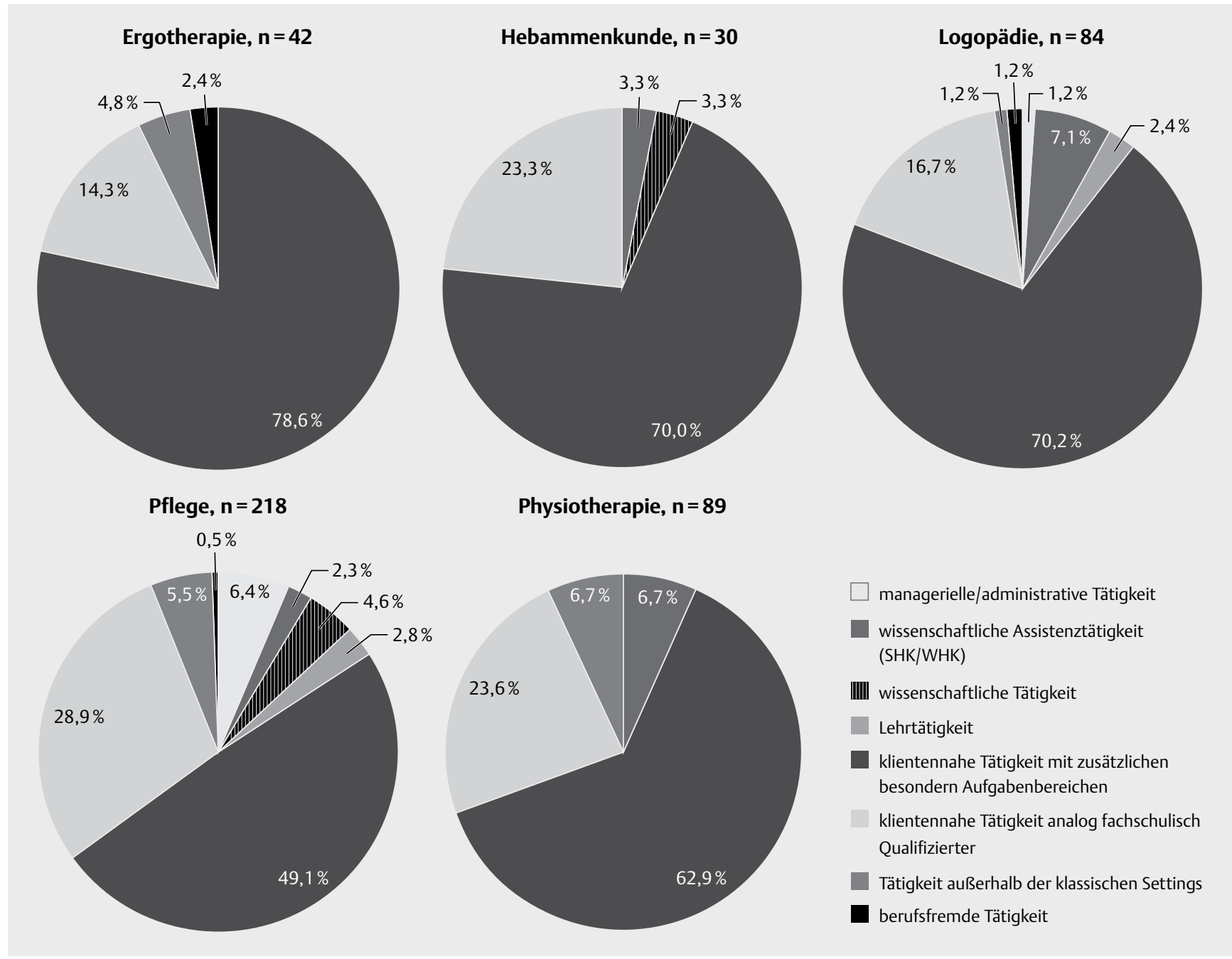

Abb. 1 Aufgabenprofile der Haupterwerbstätigkeit (N), laut Angabe der Absolventen. 


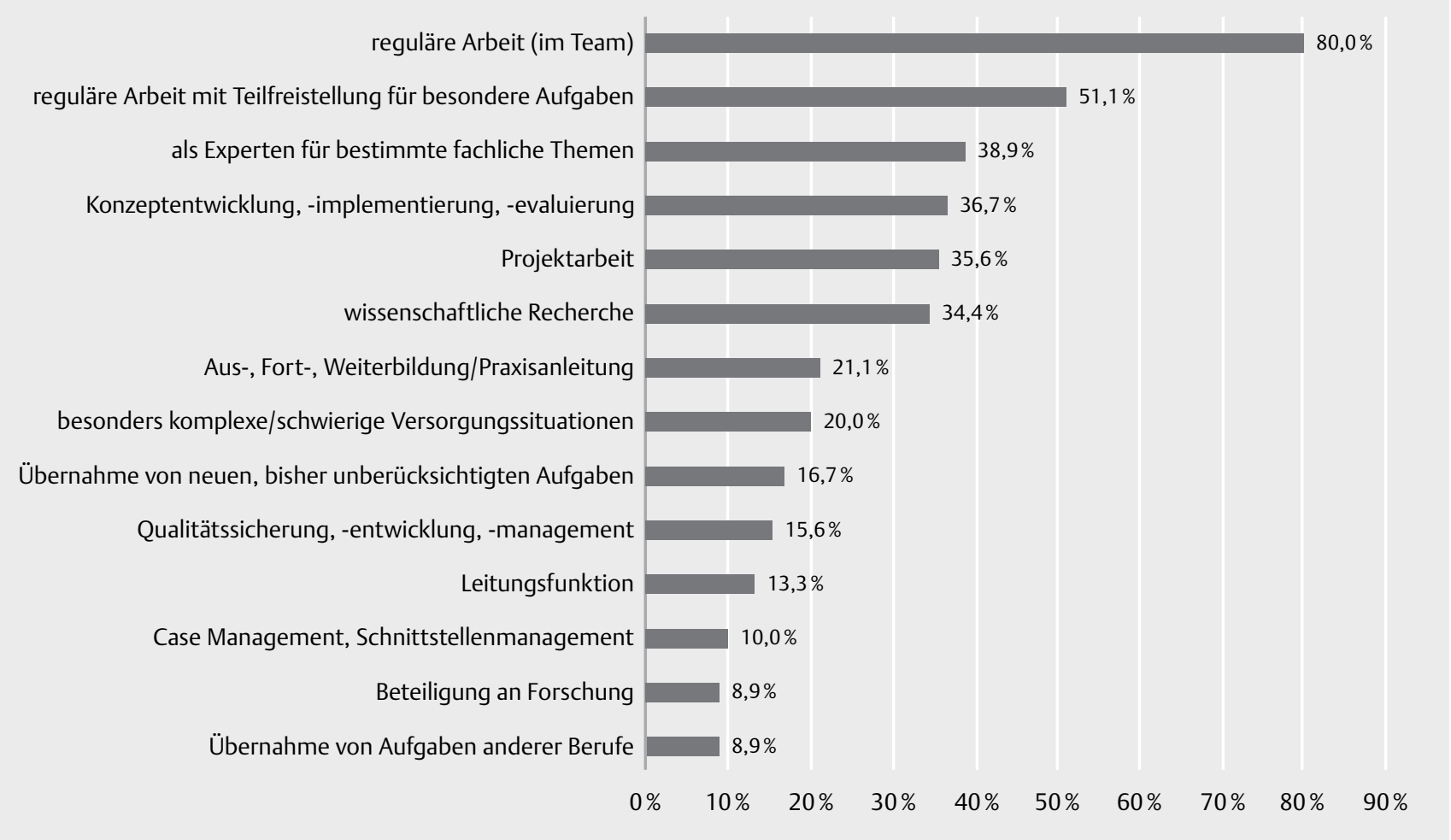

Abb. 2 Aufgaben und Verantwortungsbereiche der Absolventen aus Arbeitgebersicht [N=89] (Mehrfachnennungen waren möglich).

Ergotherapie gar nicht, in den übrigen Berufsgruppen machen diese beiden Profile jeweils zusammengenommen ca. 6-7\% aus. Vorwiegend durch Lehrtätigkeiten gekennzeichnete Profile finden sich nur in der Logopädie und der Pflege, wo sie 2,4 bzw. 2,8\% der Haupterwerbstätigkeiten ausmachen. In der Pflege spielen zudem noch Erwerbstätigkeiten mit vorwiegend manageriellen bzw. administrativen Tätigkeiten eine Rolle (6,4\%).

In allen Studienrichtungen ließ sich ein dominierendes Setting identifizieren, in dem der Großteil der Absolventen ihre Haupterwerbstätigkeiten ausübt. Unter Hebammen (53,3\%) und Pflegenden (55,8\%) ist dies das Krankenhaus, in der Ergotherapie (50,0\%), der Logopädie $(60,7 \%)$ und der Physiotherapie $(47,2 \%)$ dominieren angestellte Tätigkeiten in einer Therapiepraxis.

Nach Auskunft der Arbeitgeber finden sich die Studienabsolventen überwiegend in klientennahen Verantwortungsbereichen und oftmals auf gleichen Positionen wie fachschulisch Ausgebildete. $80,0 \%$ der Arbeitgeber setzen die Absolventen für reguläre Aufgaben ein, also solchen, die auch von fachschulisch qualifizierten Berufsangehörigen wahrgenommen werden ( $>$ Abb. 2). Über die Hälfte $(51,1 \%)$ gibt an, den Studienabsolventen zur Bearbeitung besonderer Aufgaben eine Teilfreistellung von regulären Aufgaben zu ermöglichen. Bei den besonderen Aufgaben, die sich von denen fachschulisch qualifizierter Personen unterscheiden, werden insbesondere genannt: „Expertentätigkeiten für spezifische fachliche Themen“ (38,9\%), Aufgaben bei der „Konzeptentwicklung, -implementierung und -evaluierung“ (36,7\%), sowie Aufgaben im Rahmen von „Projektarbeit“ (35,6\%). Für „wissenschaftliche Recherche“ werden die Studienabsolventen ebenfalls von gut einem Drit- tel der Arbeitgeber eingesetzt (34,4\%). Verschiedene andere Aufgaben ergänzen das Spektrum.

\section{Ausprägung und Nutzung der Kompetenzen der Absolventen}

Auf den HePCoS-Skalen zur Selbsteinschätzung der beruflichen Handlungskompetenz erreichen bzw. übertreffen alle Berufsgruppen bis auf wenige Ausnahmen im Median die Schwelle von 66,7 Punkten, was einer durchschnittlichen Einstufung von „eher sicher“ entspricht ( Tab. 1). Ausnahmen bilden in der Ergotherapie die Subskala „Praxisanleitung“ in der Kompetenzdimension „Anleiten und Schulen“ $(55,6)$ sowie die Skalen „Qualität sichern und entwickeln “ in der Logopädie, Physiotherapie (jeweils 62,5) und Hebammenkunde $(61,8)$ sowie die Skala „Wissenschaftliche Erkenntnisse recherchieren, bewerten und kommunizieren “ in der Ergotherapie $(61,9)$. Eine besonders hohe Handlungskompetenz gaben die Absolventen in der Dimension „Interprofessionell handeln“ an (76,2 bis 85,7, je nach Berufsgruppe). Auch in der Dimension „Planen, Steuern und Evaluieren therapeutischer/ pflegerischer/ hebammenkundlicher Prozesse“ wurden zwischen 73,3 und 80,0 Punkte erreicht. Der Medianscore auf der Subskala „Schulen und Anleiten von Klient/innen “ lag in der Logopädie, Physiotherapie und Pflege bei 80,0 Punkten.

Hinsichtlich der Nutzung ihrer im Bachelorstudium erworbenen Kompetenzen in der beruflichen Praxis ihrer Haupterwerbstätigkeiten unterscheiden sich die Angaben der Absolventen deutlich zwischen den Studienrichtungen ( $\triangleright$ Abb. 3). Auf einer Skala von 0 (gar nicht) bis 4 (in sehr hohem Maße) erreichten die Hebammen 
- Tab. 1 Berufliche Handlungskompetenzen (HePCoS), Selbsteinschätzung der Absolventen.

\begin{tabular}{|c|c|c|c|c|}
\hline Kompetenzdimension (Messinstrument) & $\mathbf{N}$ & Mittelwert & Median & SD \\
\hline \multicolumn{5}{|l|}{ Ergotherapie (HePCoS-T/ HePCoS-U) } \\
\hline Prozesssteuerung & 41 & 68,6 & 73,3 & 17,8 \\
\hline Durchführen klientenbezogener Interventionen & 41 & 63,2 & 66,7 & 15,4 \\
\hline Beraten und Informieren & 44 & 67,7 & 70,8 & 15,4 \\
\hline Praxisanleitung & 25 & 53,9 & 55,6 & 21,8 \\
\hline Schulen und Anleiten von Klienten & 39 & 68,5 & 66,7 & 12,2 \\
\hline Qualität sichern und entwickeln & 33 & 60,7 & 66,7 & 21,2 \\
\hline Wissenschaftliche Erkenntnisse recherchieren, bewerten und kommunizieren & 20 & 55,6 & 61,9 & 27,5 \\
\hline Interprofessionell zusammenarbeiten & 44 & 80,8 & 82,9 & 17,1 \\
\hline \multicolumn{5}{|l|}{ Logopädie (HePCoS-T/ HePCoS-U) } \\
\hline Prozesssteuerung & 83 & 76,7 & 80,0 & 15,2 \\
\hline Durchführen klientenbezogener Interventionen & 85 & 74,3 & 73,3 & 14,7 \\
\hline Beraten und Informieren & 82 & 72,8 & 70,8 & 13,4 \\
\hline Praxisanleitung & 61 & 66,2 & 66,7 & 19,9 \\
\hline Schulen und Anleiten von Klienten & 84 & 76,5 & 80,0 & 14,7 \\
\hline Qualität sichern und entwickeln & 56 & 61,6 & 62,5 & 18,2 \\
\hline Wissenschaftliche Erkenntnisse recherchieren, bewerten und kommunizieren & 57 & 64,6 & 66,7 & 20,0 \\
\hline Interprofessionell zusammenarbeiten & 76 & 80,3 & 76,2 & 17,8 \\
\hline \multicolumn{5}{|l|}{ Physiotherapie (HePCoS-T/ HePCoS-U) } \\
\hline Prozesssteuerung & 87 & 76,0 & 80,0 & 15,2 \\
\hline Durchführen klientenbezogener Interventionen & 90 & 71,3 & 72,2 & 15,9 \\
\hline Beraten und Informieren & 87 & 69,1 & 70,8 & 16,5 \\
\hline Praxisanleitung & 56 & 59,8 & 66,7 & 24,9 \\
\hline Schulen und Anleiten von Klienten & 86 & 79,2 & 80,0 & 17,4 \\
\hline Qualität sichern und entwickeln & 64 & 58,7 & 62,5 & 20,9 \\
\hline Wissenschaftliche Erkenntnisse recherchieren, bewerten und kommunizieren & 61 & 64,9 & 66,7 & 23,4 \\
\hline Interprofessionell zusammenarbeiten & 68 & 79,6 & 77,0 & 18,1 \\
\hline \multicolumn{5}{|l|}{ Hebammenkunde (HePCoS-M/ HePCoS-U) } \\
\hline Prozesssteuerung & 29 & 77,3 & 80,0 & 13,2 \\
\hline Durchführen klientenbezogener Interventionen & 29 & 72,6 & 73,3 & 17,6 \\
\hline Beraten und Informieren & 28 & 66,5 & 71,4 & 15,0 \\
\hline Praxisanleitung & 16 & 72,7 & 76,7 & 25,4 \\
\hline Schulen und Anleiten von Klienten & \multicolumn{4}{|c|}{-keine validierte Skala } \\
\hline Qualität sichern und entwickeln & 16 & 61,7 & 61,8 & 14,9 \\
\hline Wissenschaftliche Erkenntnisse recherchieren, bewerten und kommunizieren & 18 & 66,2 & 66,7 & 19,7 \\
\hline Interprofessionell zusammenarbeiten & 22 & 81,4 & 84,5 & 15,4 \\
\hline \multicolumn{5}{|l|}{ Pflege (HePCoS-N/ HePCoS-U) } \\
\hline Prozesssteuerung & 177 & 75,5 & 74,1 & 17,2 \\
\hline Durchführen klientenbezogener Interventionen & 180 & 75,5 & 77,8 & 15,9 \\
\hline Beraten und Informieren & 177 & 68,4 & 66,7 & 18,6 \\
\hline Praxisanleitung & 151 & 64,6 & 66,7 & 23,0 \\
\hline Schulen und Anleiten von Klienten & 177 & 76,9 & 80,0 & 19,1 \\
\hline Qualität sichern und entwickeln & 156 & 64,3 & 66,7 & 21,1 \\
\hline Wissenschaftliche Erkenntnisse recherchieren, bewerten und kommunizieren & 132 & 67,7 & 66,7 & 23,8 \\
\hline Interprofessionell zusammenarbeiten & 174 & 80,9 & 85,7 & 18,6 \\
\hline
\end{tabular}

ein arithmetisches Mittel von 3,25. In der Physiotherapie lag der Mittelwert bei 2,61, in der Logopädie bei 2,47, in der Ergotherapie bei 2,46 und in der Pflege bei 1,76.
In der Ergotherapie sowie in der Pflege unterscheiden sich dabei jene Absolventen mit Aufgabenprofilen analog fachschulisch qualifizierter Kollegen von Absolventen, deren vorwiegend klientennahe Tätigkeit zusätzlich um „besondere“ Aufgabenbereiche er- 
Ergotherapie ( $n=41$ Haupterwerbstätigkeiten) $(M W=2,5)$

Logopädie ( $\mathrm{n}=83$ Haupterwerbstätigkeiten) $(\mathrm{MW}=2,5)$

Physiotherapie ( $\mathrm{n}=89$ Haupterwerbstätigkeiten)

$(\mathrm{MW}=2,6)$

Hebammenkunde ( $\mathrm{n}=28$ Haupterwerbstätigkeiten)

$(\mathrm{MW}=3,3)$

Pflege ( $n=214$ Haupterwerbstätigkeiten) $(M W=1,8)$
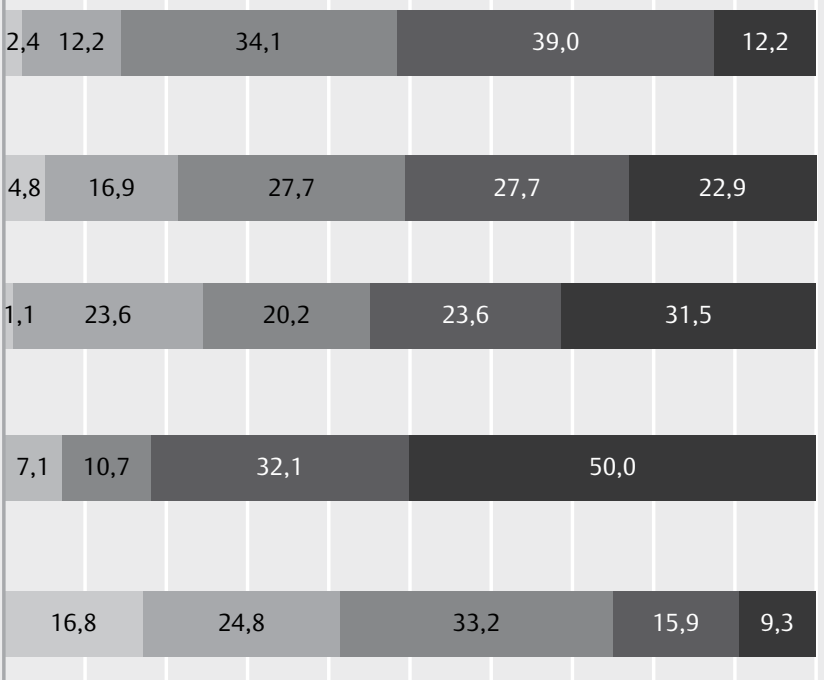

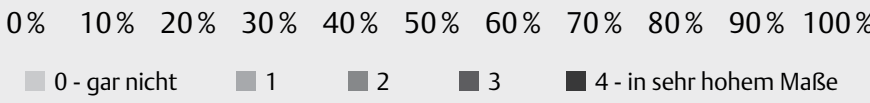

Abb. 3 Nutzung der im Studium erworbenen Kompetenzen im Rahmen der Haupterwerbstätigkeit (nach Studienrichtung), laut Angabe der Absolventen.

- Tab. 2 Nutzung der im Studium erworbenen Kompetenzen im Rahmen der Haupterwerbstätigkeit in den 2 vorwiegend klientennahen Aufgabenprofilen, laut Angabe der Absolventen.

\begin{tabular}{|c|c|c|c|c|c|c|c|}
\hline Studienrichtung & Aufgabenprofil & $\mathbf{N}$ & MW & \multicolumn{2}{|c|}{ KI $95 \%$} & SD & $\mathbf{p}$ \\
\hline \multirow[t]{2}{*}{ Ergotherapie } & analog fachschulisch Qualifizierter & 5 & 1,60 & 0,92 & 2,28 & 0,548 & \multirow[t]{2}{*}{${ }^{*} 0,014$} \\
\hline & besondere zusätzliche Aufgabenbereiche & 33 & 2,67 & 2,36 & 2,97 & 0,854 & \\
\hline \multirow[t]{2}{*}{ Logopädie } & analog fachschulisch Qualifizierter & 13 & 2,15 & 1,34 & 2,97 & 1,345 & \multirow[t]{2}{*}{0,266} \\
\hline & besondere zusätzliche Aufgabenbereiche & 59 & 2,56 & 2,27 & 2,85 & 1,118 & \\
\hline \multirow[t]{2}{*}{ Physiotherapie } & analog fachschulisch Qualifizierter & 21 & 2,62 & 2,05 & 3,19 & 1,244 & \multirow[t]{2}{*}{0,887} \\
\hline & besondere zusätzliche Aufgabenbereiche & 56 & 2,57 & 2,25 & 2,89 & 1,204 & \\
\hline \multirow[t]{2}{*}{ Hebammenkunde } & analog fachschulisch Qualifizierter & 6 & 3,50 & 2,93 & 4,00 & 0,548 & \multirow[t]{2}{*}{0,656} \\
\hline & besondere zusätzliche Aufgabenbereiche & 20 & 3,15 & 2,66 & 3,64 & 1,040 & \\
\hline \multirow[t]{2}{*}{ Pflege } & analog fachschulisch Qualifizierter & 60 & 1,22 & 0,93 & 1,50 & 1,106 & \multirow[t]{2}{*}{$* 0,004$} \\
\hline & besondere zusätzliche Aufgabenbereiche & 106 & 1,70 & 1,49 & 1,90 & 1,062 & \\
\hline
\end{tabular}

Wertebereich: 0 (gar nicht) bis 4 (in sehr hohem Maße). ${ }^{*}$ signifikant auf $\alpha=0,05-$ Niveau (Mann-Whitney-U-Tests).

weitert ist ( $>$ Tab. 2). Letztere weisen in den beiden Berufsgruppen signifikant höhere Mittelwerte für die Nutzung der im Studium erworbenen Kompetenzen in ihrer beruflichen Praxis auf (Pflege: $p=0,004$; Ergotherapie: $p=0,014$, Mann-Whitney-U-Test). Dieser Unterschied besteht auch, wenn neben den Haupterwerbstätigkeiten die Nebenerwerbstätigkeiten mit einbezogen werden. Allerdings ist in der Ergotherapie die geringe Fallzahl von Absolventen mit dem Profil „klientennah analog fachschulisch Qualifizierter“ zu beachten.

Die Ergebnisse der Befragung der Arbeitgeber zeigen, dass aus ihrer Sicht die Kompetenzen der Absolventen in der beruflichen Praxis erkennbar sind. In allen 7 abgefragten Kompetenzdimensionen trifft dies jeweils für die große Mehrheit (jeweils rund 84 bis $93 \%)$ der Arbeitgeber „voll“ oder „eher“ zu.
Im Vergleich mit fachschulisch Qualifizierten hinsichtlich unterschiedlicher Ausprägungen der Kompetenzdimensionen wird deutlich, dass die befragten Arbeitgeber einen Vorteil aufseiten der Absolventen feststellen. Sehr deutliche Vorteile bei den Absolventen werden bei Wissenschaftlichkeit, Prozesssteuerung und Qualitätssicherung gesehen, in denen die Kompetenzen von zwischen 69,5\% bis $91,4 \%$ der Arbeitgeber als stärker ausgeprägt eingeschätzt werden. Rund die Hälfte der Befragten erkennt ausgeprägtere Kompetenzen der Absolventen in den Bereichen Beratung, interprofessionelle Kooperation und Anleitung, während die andere Hälfte beide Gruppen gleich einschätzt. Lediglich bei der Durchführung klientenbezogener Maßnahmen schätzt eine Mehrheit der Arbeitgeber beide Gruppen gleich ein und es findet sich auch der einzig nennenswerte Anteil an Befragten, die die Kompetenzen der fachschu- 


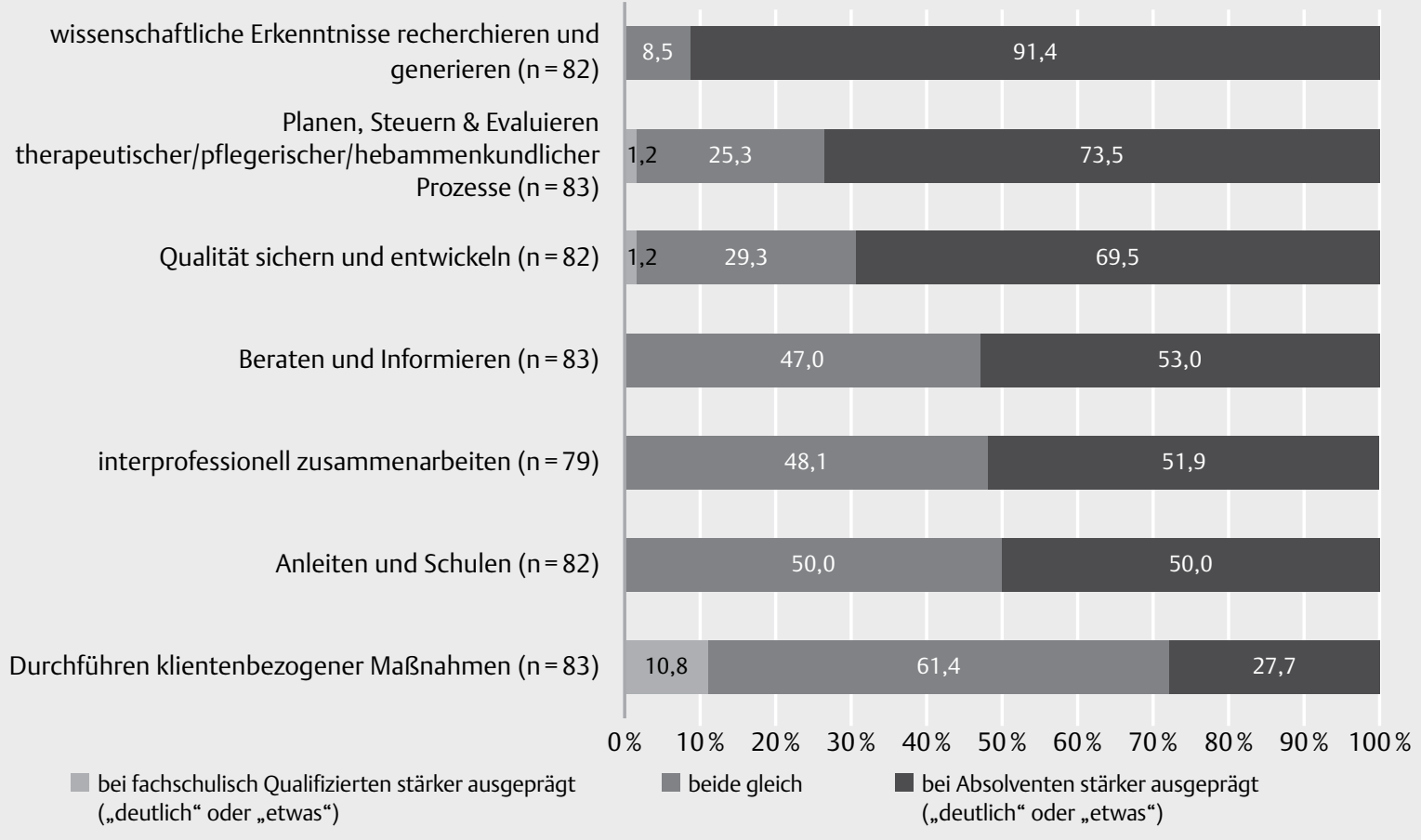

Abb. 4 Unterschiede der Kompetenzen bei Absolventen und fachschulisch Qualifizierten aus Arbeitgebersicht.

lisch ausgebildeten Beschäftigten als stärker ausgeprägt einschätzen ( $\triangleright$ Abb. 4).

Ergänzend zu den aus den „gemeinsamen Qualifizierungszielen" abgeleiteten Kompetenzdimensionen wurden weitere Merkmale abgefragt, bei denen die Fähigkeiten der Absolventen in der beruflichen Praxis im Vergleich zu fachschulisch ausgebildeten Personen insgesamt positiv eingeschätzt werden. Insbesondere bei den Merkmalen Evidenzbasierung, Analyse- und Reflexionsvermögen, Aufstiegsorientierung und kritischem Hinterfragen werden die Absolventen als deutlich stärker wahrgenommen. Bei den weiteren Merkmalen werden beide Gruppen mehrheitlich als gleich eingeschätzt, wobei jeweils die Absolventen im Vergleich bessere Werte erreichen als die fachschulisch Qualifizierten. Lediglich bei den „praktischen Fertigkeiten“ ist dies nicht der Fall; hier werden die fachschulisch Ausgebildeten als stärker bewertet.

97,6\% der befragten Arbeitgeber äußern sich („voll“ und „eher“) zufrieden mit den Kompetenzen der eingestellten Absolventen. Dass die Kompetenzen den Anforderungen der Berufspraxis entsprechen, finden $90,3 \%$, wobei hier lediglich $15,9 \%$,voll“ zustimmen. Die Befragten sind zudem zu 87,2\% der Ansicht, dass sich die akademische Grundqualifikation bewährt. Dass die hochschulisch Qualifizierten einen zusätzlichen Beitrag zur Versorgung leisten können, meinen 88,4\% der Befragten.

\section{Diskussion}

Die hier betrachteten berufsqualifizierenden Modellstudiengänge werden in erster Linie als Antwort auf die Herausforderungen in der Versorgungspraxis gesehen [1,2]. Primäres Ziel ist es, die Absol- venten für klientennahe Tätigkeiten in Arbeitsfeldern der direkten Versorgung zu qualifizieren. Um die Konformität und den Erfolg der Studiengänge im Hinblick auf dieses Ziel einschätzen zu können, wurden die Arbeitsfelder und Aufgabenbereiche der Absolventen analysiert. Die dominierenden Arbeitsfelder therapeutische Praxis und Krankenhaus entsprechen weitgehend denen Berufsangehöriger mit fachschulischer Ausbildung [18]. Ein professionsadäquater Verbleib der Studienabsolventen wird darüber hinaus durch die empirischen Befunde aus den fachspezifischen bundesweiten Untersuchungen zum Verbleib von Modellstudiengangabsolventen bestätigt $[11,12,13,14]$. Auch hier wird beschrieben, dass die Absolventen vornehmlich in der klientennahen Versorgung verbleiben, mit einem etwas geringeren Anteil in der Pflege. Dies kann als Hinweis auf eine höhere Bindung der therapeutischen und hebammenkundlichen Absolventen an den klientenahen Bereich gedeutet werden. Auch für Logopädieabsolventen eines additiven Bachelorstudiengangs wurde z. B. festgestellt, dass diese größtenteils als Angestellte in Therapiepraxen (weiter-)arbeiten [19]. Gleichsam ist es möglich, dass der Befund auf diversifiziertere Beschäftigungsmöglichkeiten für Pflegeakademiker zurückzuführen ist, die in der Vergangenheit bereits in Folge verschiedener additiver Studiengangformen in den Bereichen Pflegewissenschaft, -management und -pädagogik entstanden sind [20,21]. Hierdurch gab es in der Pflege eher Möglichkeiten, auch in klientenfernen Arbeitsfeldern tätig zu werden. Im Gegensatz zu Absolventenbefragungen additiver Studiengangformate [20], zeigt sich aber auch bei den Pflegenden in VAMOS nur eine sehr geringe Tendenz, in klientenfernen Settings zu arbeiten. Annahmen, dass die Absolventen nach einem Modelstudium vorwiegend in Arbeitsfeldern in Wissenschaft, Lehre 
oder Management tätig sein könnten [22], werden in keiner Berufsgruppe bestätigt. Das berufsqualifizierende Studienformat scheint also, wie vom Gesetzgeber vorgesehen, vornehmlich ein Wegbereiter für Berufslaufbahnen innerhalb des klientennahen Tätigkeitsspektrums zu sein. Die Dauer des Verbleibs in diesem Bereich ist aufgrund der bislang zu kurzen Berufstätigkeit schwer einzuschätzen. Insbesondere die Verbesserung der Vergütungssituation und die Anwendung der hochschulisch erworbenen Kompetenzen scheinen hier wesentliche Faktoren für den längerfristigen Verbleib zu sein $[10,11,12,13]$. Die festgestellten Aufgabenbereiche können dahingehend interpretiert werden, dass sich die postulierte Ausweitung der Aufgabenprofile und des Kompetenzspektrums für hochschulisch Qualifizierte [1, 2] in der Berufspraxis andeutet. Von Absolventen- und Arbeitgeberseite werden Aufgabenerweiterungen im Vergleich zu fachschulischen Kollegen beschrieben, die sowohl als Indiz für eine wissenschaftlich-fachliche Vertiefung (u.a. Aufgaben als „Expertin bzw. Experte für bestimmte fachliche Themen “ und „wissenschaftlichen Recherche") als auch für eine überfachliche Verbreiterung (u.a. „Konzeptentwicklung, -implementierung und -evaluierung“, „Projektarbeit“) der Kompetenzen durch das Studium gedeutet werden können. Der mit den Studienprogrammen angestrebte Kompetenzzugewinn, der den Fokus auf die „Wissenschaftsbasierung“ und auf die „institutionelle und gesundheitssystemische Gestaltung“ legt [23], spiegelt sich in der Berufspraxis in unterschiedlichen Aufgabenprofilen von Absolventen und fachschulisch qualifizierten Kollegen wider. Dies scheint Erfahrungen aus europäischen Vergleichsländern zu bestätigen, wo die Übernahme erweiterter Aufgaben und Rollen im Zuge der Akademisierung festzustellen ist [24]. Hierzulande sind die Erweiterungen zwar bisher eher Folge individueller Aushandlungsprozesse und weniger als Folge existierender Stellenprofile zu deuten; allerdings kann aus der Anzahl an Betrieben, die eine (Teil-)Freistellung einräumen, geschlussfolgert werden, dass Ansätze einer betrieblichen Einbindung der Absolventen erkennbar werden. Die Möglichkeiten von job enlargement (Ausweitung des Tätigkeitsfelds durch weitere Aufgaben auf vergleichbarem Anforderungsniveau) und job enrichment (Anreicherung des Tätigkeitsfelds um höherwertige Aufgaben, i.d.R. in Verbindung mit zusätzlichen Entscheidungsbefugnissen) für hochschulisch Qualifizierte [23] deuten sich an. Im Hinblick auf die stärkere Nutzung hochschulisch erworbener Kompetenzen sind diese Möglichkeiten vermutlich v. a. in der Pflege eine wichtige Stellschraube. Die Selbsteinschätzung der Kompetenzen deutet in allen Dimensionen auf eine ausgeprägte Handlungssicherheit der Absolventen in der Berufspraxis hin. In besonders hohem Maße gilt dies für die Dimension der interprofessionellen Zusammenarbeit. Der Befund korrespondiert mit den Ergebnissen anderer Untersuchungen zu den Modellstudiengängen $[8,12,33]$ und spricht für eine erfolgreiche Vermittlungspraxis. Die Fremdeinschätzung durch die befragten Arbeitgeber zeigt, dass die große Mehrheit insgesamt positive Auswirkungen für die Versorgungssituation durch die hochschulisch erworbenen Kompetenzen vermutet. Die Arbeitgeber bestätigen eine gute Passung mit den Anforderungen der Berufspraxis, was auf eine adäquate Beschäftigungsfähigkeit der Absolventen schließen lässt. Vor dem Hintergrund der gesetzlichen Verpflichtung, mit Bachelorstudiengängen v. a. für die Beschäftigungsfähigkeit zu sorgen [25], wird ein wesentliches Bildungsziel der Studiengänge erreicht. Den be- deutsamsten Kompetenzvorsprung im Vergleich zu fachschulisch Qualifizierten nehmen die befragten Arbeitgeber beim Recherchieren und Nutzen wissenschaftlicher Erkenntnisse, bei der Prozesssteuerung, und bei der Qualitätssicherung und -entwicklung wahr. Am geringsten scheint der Kompetenzzugewinn durch das Studium bei der Durchführung klientenbezogener Maßnahmen. Hier sollte berücksichtigt werden, dass die Einschätzung der Arbeitgeber vor dem Hintergrund einer erst kurzen Berufstätigkeit der Absolventen gegeben wurde. Die aktuellen Befunde der Kompetenzanalyse stehen insgesamt im Einklang mit den Erkenntnissen aus der ersten Evaluationsphase. Die Vermittlung der den Studiengangzielen entsprechenden Kompetenzen gelingt [8]. Die Ergebnisse zur Nutzung der Kompetenzen in der Berufspraxis deuten allerdings darauf hin, dass eine qualifikationsadäquate Beschäftigung in der klientennahen Versorgung nicht immer erfolgt. Die Herausforderung bei der Integration der hochschulischen Qualifikation z. B. in der Pflege deckt sich mit anderen Befunden aus dem Berufsfeld, die Defizite bei der Aufgabenbeschreibung und Kompetenzzuordnung für hochschulisch qualifizierte Pflegende feststellen [20, 21, 24]. Insbesondere für diese Berufsgruppe scheint es bedeutsam, die Aufgabenprofile zu erweitern, um die erworbenen Kompetenzen adäquat einzusetzen. In den anderen Berufsgruppen erscheint dies weniger relevant. Dies könnte zum einen in der Ausrichtung der Studienmodelle begründet sein. Anders als in der Pflege sind die Modelle im therapeutischen und hebammenkundlichen Bereich weniger auf eine Erweiterung der Aufgaben ausgerichtet, sondern vielmehr auf die Qualitätsverbesserung der professionstypischen Tätigkeiten [1]. Zum anderen ist die unterschiedliche Bedeutung vermutlich auch berufsgruppenspezifischen Besonderheiten der Arbeitssettings geschuldet. Obwohl Hebammen, wie Pflegende, vorwiegend im Arbeitssetting Krankenhaus tätig sind, besteht schon länger durch Vorbehaltsaufgaben eine traditionell große Handlungsautonomie, die sich vermutlich förderlich auf die Kompetenznutzung auswirkt. In den therapeutischen Berufen wiederum ermöglicht das stark dominierende Arbeitssetting der ambulanten Therapiepraxis im Vergleich zum Krankenhaus einen weniger engen institutionellen Rahmen [26], was vermutlich zur individuellen Nutzung der hochschulisch erworbenen Kompetenzen beitragen kann. Mit Ausnahme der Hebammen machen die Ergebnisse in allen Berufsgruppen deutlich, dass das Kompetenzpotenzial der Absolventen der berufsqualifizierenden Modellstudiengänge noch nicht optimal ausgeschöpft wird.

\section{Limitationen der Studie}

Die Erfassung der Kompetenzen beruht auf der Selbsteinschätzung der Absolventen und einer Bewertung durch die Arbeitgeberseite. Kompetenzunterschiede zu fachschulisch ausgebildeten Kollegen werden daher vor dem Hintergrund einer relativ kurzen Zeit der Hochschulabsolventen im Beruf und des Fehlens einer Vergleichsgruppe aus äquivalenten Fachschulabsolventen festgestellt. Bei dieser Form der Kompetenzerfassung können Verzerrungen nicht ausgeschlossen werden. Vor dem Hintergrund der Orientierungsund Bewährungszeit von Berufseinsteigern sind Unterschiede zu fachschulisch Ausgebildeten, die schon lange im Beruf sind, schwer zu erheben und es ist wahrscheinlich, dass die erworbenen Kompetenzen der Hochschulabsolventen zum Zeitpunkt der Querschnittsbefragung noch nicht vollumfänglich zum Tragen kommen. 
Inwieweit die befragten Arbeitgeber alle potenziellen Arbeitgeber repräsentieren, kann darüber hinaus nicht beurteilt werden. Für eine systematische Beeinflussung der Ergebnisse liegen keine Hinweise vor, letztlich kann dies aber nicht ausgeschlossen werden. Eine realistische Abschätzung der Grundgesamtheit war bei den Arbeitgebern nicht möglich.

\section{Schlussfolgerungen}

Die Ergebnisse stützen die empfohlene Überführung der Studiengänge in eine regelhafte hochschulische Erstqualifizierung [8] in den untersuchten Berufsgruppen Pflege, Hebammenkunde, Physiotherapie, Logopädie und Ergotherapie. Die mit den Studiengängen verbundenen Bildungsziele werden erreicht. Ein Kompetenzzugewinn im Vergleich zur fachschulischen Ausbildung wird in der Berufspraxis besonders in den Bereichen des wissenschafts- und evidenzbasierten Handelns und in der kooperativen Zusammenarbeit erkennbar. Die zur Stärkung der Versorgung getätigten Bildungsinvestitionen erzielen den intendierten Effekt. Es erscheint daher folgerichtig, dass ein Studium in der Pflege als reguläre Ausbildungsform ergänzend zur beruflichen Ausbildung gesetzlich verankert wurde. In der Hebammekunde ersetzt das Studium die fachschulische Ausbildung mittelfristig sogar vollständig. Auch in den Therapieberufen sollten nun schnellstmöglich die berufsgesetzlichen Regelungen angepasst werden, damit der im internationalen Vergleich zögerliche Akademisierungs- und Professionalisierungsprozess forciert wird und die Hochschulen Planungssicherheit für die Etablierung entsprechender Programme erhalten. Die Anforderungs- und Aufgabenprofile für hochschulisch Qualifizierte sollten hierbei in einem engen Austausch von Wissenschaft und Versorgungspraxis aufgegriffen und weiter konkretisiert werden. Bei der adäquaten inhaltlichen Ausgestaltung der Praxisanteile ist hierbei nicht nur die Quantität, sondern insbesondere auch die Qualität des Kompetenzerwerbs zu berücksichtigen (z. B. realitätsnahe Simulationsszenarien in Skills-Labs, Projektseminare und Lehrangebote mit Klientenkontakt). Um den festgestellten Kompetenzzugewinn für die Versorgungspraxis besser nutzbar zu machen, sind zudem Veränderungen der Organisations- und Versorgungsstrukturen notwendig. Mehr Stellenprofile mit qualifikationsadäquaten Handlungsspielräumen, die eine eigenverantwortlichere und flexiblere Versorgung ermöglichen, sollten geschaffen werden. Entsprechende Modelle, die hierbei Vorbildfunktion haben könnten, finden sich im europäischen Ausland (u. a. die physiotherapeutische Leistungserbringung im Direktzugang sowie die Erweiterung fachspezifischer Handlungskompetenzen von Pflegenden als „advanced practitioners“) [24] und an deutschen Unikliniken [21]. Arbeitgeber und Politik sind hier gefordert, Rahmenbedingungen herzustellen, die eine qualifikationsadäquate Tätigkeit und Vergütung für eine klientenorientierte Versorgung fördern.

\section{Interessenkonflikt}

Mit einer Ausnahme sind die Autoren Mitglieder der Hochschulen, deren Absolventenverbleib im Rahmen der Studie evaluiert wurde.

\section{Literatur}

[1] Deutscher Wissenschaftsrat (WR). Empfehlungen zu hochschulischen Qualifikationen für das Gesundheitswesen. (13.07.2012). Im Internet: www.wissenschaftsrat.de/download/archiv/2411-12.pdf; Stand: 27.02.2020

[2] Sachverständigenrat zur Begutachtung der Entwicklung im Gesundheitswesen (SVR). Koordination und Integration - Gesundheitsversorgung in einer Gesellschaft des längeren Lebens. Sondergutachten 2009. Kurzfassung (05.2009). Im Internet: https://www.svr-gesundheit.de/fileadmin/user_upload/Gutachten/2009/Kurzfassung-2009. pdf; Stand: 27.02 .2020

[3] Walkenhorst U, Mahler C, Aistleithner R et al. Position statement GMA Comittee - "Interprofessional Education for the Health Care Professions”. GMS Zeitschrift für Medizinische Ausbildung 2015; 32: 1-19

[4] Borgetto B, Räbiger J, Rottenecker J et al. Akademisierung: Die vollständige hochschulische Ausbildung in der Ergotherapie. Logopädie und Physiotherapie ist notwendig und machbar. physioscience 2019; 15: 41-42. doi:10.1055/a-0832-9668

[5] Gesetz über die Berufe in der Krankenpflege (Krankenpflegegesetz KrPflG). In der Fassung vom 16.07.2003 (BGBI. I S. 1442), zuletzt geändert durch Artikel 1a des Gesetzes vom 17. Juli 2017 (BGBI. IS. 2581). (21.07.2003). Im Internet: https://www.bgbl.de/xaver/bgbl/ start.xav?start $=\% 2 \mathrm{~F} \% 2 \mathrm{~F} * \% 5 \mathrm{~B} \% 40 \mathrm{attr}$ id \%3D \%27bgbl103s 1442 . pdf\%27\%5D\#_bgbl_\%2F\%2F *5B \%40attr_id \%3D \%27bgbl103s1442.pdf\%27\%5D_1582797033976; Stand: 27.02 .2020

[6] Gesetz über die Berufe in der Altenpflege (Altenpflegegesetz - AltPflG). In der Fassung vom 25. August 2003 (BGBI. I S. 1690), zuletzt geändert durch Artikel 1b des Gesetzes vom 17. Juli 2017 (BGBI. I S. 2581) (04.09.2003). Im Internet: https://www.bgbl.de/xaver/bgbl/ start.xav?start= // \%5B@attr_id =\%27bgbl103s1690.pdf\%27\%5D\#_ bgbl__\%2F\%2F \%5B \%40attr_id\%3D\%27bgbl103s1690. pdf\%27\%5D_1582797378103; Stand: 27.02.2020

[7] Gesetz zur Einführung einer Modellklausel in die Berufsgesetze der Hebammen, Logopäden, Physiotherapeuten und Ergotherapeuten. In der Fassung vom 25.09.2009 (BGBI I S.3158) (02.10.2009). Im Internet: https://www.bgbl.de/xaver/bgbl/start.xav?startbk= Bundesanzeiger_BGBI\&start= // \%5B@attr_id=\%2527bgbl109s3158. pdf\%2527\%5D\#_bgbl_\%2F\%2F *\%5\%40attr_id \%3D\%27bgbl109s3158.pdf\%27\%5D_1561471479049; Stand: 27.02 .2020

[8] Darmann-Finck I, Reuschenbach B. Ergebnisse der Evaluation der hochschulischen Erstausbildung in den therapeutischen Berufen. Gesundheitswesen 2019; 81: 325-331. doi:10.1055/s-0043-119078

[9] Darmann-Finck I, Muths S, Görres S et al. Abschlussbericht. Inhaltliche und strukturelle Evaluation der Modellstudiengänge zur Weiterentwicklung der Pflege- und Gesundheitsfachberufe in NRW. (12.2014). Im Internet: https://www.mags.nrw/sites/default/files/asset/ document/pflege_abschlussbericht_26_05_2015.pdf; Stand: 27.02.2020

[10] Dieterich S, Hoßfeld R, Latteck ÄD et al. Verbleibstudie der Absolventinnen und Absolventen der Modellstudiengänge in Nordrhein-Westfalen (VAMOS) - Abschlussbericht (04.11.2019). Im Internet: www. hs-gesundheit.de/vamos-bericht; Stand: 27.02.2020

[11] Baumann AL, Kugler C. Berufsperspektiven von Absolventinnen und Absolventen grundständig qualifizierender Pflegestudiengänge - Ergebnisse einer bundesweiten Verbleibstudie. Pflege 2018; 32: 7-16. doi:10.1024/1012-5302/a000651

[12] Blümke C, Räbiger ], Hansen $\mathrm{H}$ et al. Berufstätigkeit und Berufszufriedenheit von Therapeuten mit hochschulischer Ausbildung - Ergebnisse der HVG-Absolventenbefragung zur Evaluation von primärqualifizierenden Bachelorstudiengängen für therapeutische Gesundheitsfachberufe (04.2019). Im Internet: https://www.hv-gesundheitsfachberufe. de/wp-content/uploads/HVG-Forschungsbericht_Homepage.pdf. Stand: 27.02 .2020 
[13] Räbiger J, Rottenecker J, Lauer $\mathrm{N}$ et. al. Therapeuten mit berufsqualifizierendem Studienabschluss: Berufseinmündung und Erfahrungen im Berufsalltag - Ergebnisse einer Absolventenbefragung. Ergoscience 2019; 14: 147-155. doi.org/10.2443/skv-s-2019-54020190402

[14] Stertz N, Blättner B. Beruflicher Verbleib der ersten Absolventenkohorte des Studienganges Hebammenkunde B. Sc. an der Hochschule Fulda. (07/2017). Im Internet: https://www.hs-fulda.de/fileadmin/ user_upload/FB_Pflege_und_Gesundheit/Forschung; Stand: 27.02.2020

[15] Igl, G. Beruferechtliche Begleitforschung zur Auswertung der im Land Nordrhein-Westfalen gemäß der Verordnung über die Durchführung von Modellvorhaben zur Weiterentwicklung der Berufe in der Altenund Krankenpflege, für Hebammen, Logopäden, Ergotherapeuten und Physiotherapeuten vom 25. Februar 2010 (GV. NRW 2010, 177) durchgeführten Modellvorhaben nach $\S 4$ Absatz 6 und 7 des Altenpflegegesetzes, $\S 4$ Absatz 6 und 7 des Krankenpflegegesetzes, $\S$ 4 Abs. 5 des Ergotherapeutengesetzes, $\S 6$ Absatz 3 des Hebammengesetzes, § 4 Absatz 5 des Logopädengesetzes und § 9 Absatz 2 des Masseur- und Physiotherapeutengesetzes. Hamburg. Düsseldorf: MAGS NRW. (02/2015). Im Internet: https://www.mags.nrw/sites/ default/files/asset/document/pflege_beruferechtliche-begleitforschung.pdf; Stand: 18.05 .2020

[16] Grebe C, Schürmann M, Latteck ÄD. Die HePCoS Skalen zur Kompetenzerfassung in den Gesundheitsfachberufen. Berichte aus Lehre und Forschung Nr. 48. Bielefeld: Fachhochschule Bielefeld [im Druck]

[17] Alesi B, Neumeyer S. Studium und Beruf in Nordrhein-Westfalen. Studienerfolg und Berufseinstieg der Absolventinnen und Absolventen des Abschlussjahrgangs 2014 von Fachhochschulen und Universitäten. 2017; Im Internet: https://nbn-resolving.org/urn:nbn:de:0168ssoar-56323-8; Stand: 27.02.2020

[18] Barzel A, Ketels G, Schön G et al. Erste deutschlandweite Befragung von Physio- und Ergotherapeuten zur Berufssituation physioscience 2011; 7: 55-62. doi:10.1055/s-0031-1273376
[19] Hahn V. Was brachte uns das Studium? Eine Absolventenbefragung des Studienganges für Logopädie der HAWK Hildesheim. Forum Logopädie 2008; 22: 30-33

[20] Zieher J, Ayan T. Karrierewege von Pflegeakademikern - Ergebnisse einer bundesweiten Absolventenbefragung zu Ausbildung, Studium und Beruf. Pflege und Gesellschaft 2016; 21: 47-63

[21] Tannen A, Feuchtinger J, Strohbücker B. Survey zur Einbindung von Pflegefachpersonen mit Hochschulabschlüssen an deutschen Universitätskliniken - Stand 2015. ZEFQ 2016; 120: 39-46

[22] Gerst T. Akademisierung der Gesundheitsfachberufe: Keine Qualifizierung vom Bett weg. Dtsch Arztebl International 2013; 110: 1844

[23] Darmann-Finck I, Reuschenbach B. Qualität und Qualifikation: Schwerpunkt Akademisierung der Pflege. In Jacobs K, Kuhlmey A, Greß et al., Hrsg. Pflege-Report 2018. Qualität in der Pflege. Berlin und Heidelberg: Springer; 2018: 163-170

[24] Lehmann Y, Beutner K, Karge K et al. Bestandsaufnahme der Ausbildung in den Gesundheitsfachberufen im europäischen Vergleich (GesinE)., Hrsg. Bundesministerium für Bildung und Forschung. Bonn/ Berlin: BMBF; 2014. Im Internet: https://www.bmbf.de/upload_filestore/pub/berufsbildungsforschung_band_15.pdf; Stand: 27.02.2020

[25] Gesetz über die Hochschulen des Landes Nordrhein-Westfalen (Hochschulgesetz - HG). Vom 16. September 2014 ( $\S \S 3$ und 5) (01.2015). Im Internet: https://www.mkw.nrw/system/files/media/ document/file/mkw_nrw_hochschulgesetz-nrw.pdf; Stand: 27.02.2020

[26] Ulrich G, Homberg A, Karstens S et al. Die Arbeitszufriedenheit von Berufseinsteigern in den Gesundheitsberufen. Das Gesundheitswesen 2019; 81: 99-105. doi:10.1055/s-0043-107873 